\title{
ADENOCARCINOMA OF THE LUNG WITH RARE INSERTION MUTATION IN EGFR EXON 19 THAT HAD PARTIAL RESPONSE TO GEFITINIB: A CASE REPORT
}

\author{
V. Kozlov', I. Karpov', S. Kovalenko ${ }^{2,3}$, V. Shamanin ${ }^{2,3, ~ * ~}$ \\ ${ }^{1}$ Thoracic Department, Regional Cancer Hospital, Novosibirsk 630108, Russia \\ ${ }^{2}$ BioLink Ltd, Novosibirsk 630055, Russia \\ ${ }^{3}$ Institute of Molecular Biology and Biophysics, Novosibirsk 630117, Russia
}

\begin{abstract}
Aim: Classic activating mutations L858R and deletions in exon 19 (19del) in the gene for epidermal growth factor receptor (EGFR) are associated with sensitivity of the non-small cell lung cancer (NSCLC) to therapy with tyrosine kinase inhibitors (TKI). Insertions in EGFR exon 19 (19ins) are rare mutations in NSCLC; response of cases with 19ins to TKI is not well studied. Here we report a case of NSCLC with 19ins in a Russian patient who was treated with gefitinib. We also overview cases of 19ins reported in the literature. Case description: A 48 years old female Russian patient was diagnosed with adenocarcinoma of the lung (T3N2M1, stage IV). Mutation 19ins was detected in the tumor biopsy by fragment analysis and genotyped by Sanger sequencing as p.I744_K745insKIPVAI. Treatment with gefitinib $(250 \mathrm{mg} /$ day $)$ resulted in clinical and radiological improvements scored as partial response that lasted 12 months. Conclusion: Treatment with gefitinib of lung adenocarcinoma that carries mutation EGFR 19ins can result in durable response.
\end{abstract}

Key Words: lung adenocarcinoma, EGFR, exon 19 insertion, gefitinib.

Some cases of non-small cell lung cancer (NSCLC) carry activating mutations in the epidermal growth factor receptor (EGFR) gene which are associated with sensitivity of the tumor to tyrosine kinase inhibitors (TKI). Frequency of the mutations varies from 2 to $40 \%$ in different groups of patients and positively correlates with adenocarcinoma histology, female gender, non-smoker status and Asian ethnicity [1]. Most of activating mutations in EGFR gene (85-90\%) are small in-frame deletions in exon 19 (19del), or missense mutation L858R. Insertions in EGFR exon 19 (19ins) are rare mutations which comprise about $1 \%$ among cases of NSCLC with mutant EGFR [2]. Due to the small number of patients response to TKI of cases with 19ins is not well studied. Here we describe a case of NSCLC with 19ins in a Russian patient that was treated with gefitinib.

\section{CASE REPORT}

Female Caucasian patient, 48 years old, nonsmoker, was diagnosed with lung cancer (T3N2M1, stage IV). Major clinical symptoms were pronounced dyspnea and cough with large amount of sputum (up to $500 \mathrm{ml} /$ day). Computed tomography (CT) scans showed peripheral tumor of the right lung (Fig. 1, a). Thoracoscopic biopsy of the right lung was performed to verify morphological diagnosis. Biopsy of the tumor had histology of adenocarcinoma.

Molecular tests of the DNA from FFPE tumor biopsy did not find common mutations EGFR L858R or 19del. The assays we used are based on allele-specific realtime polymerase chain reaction (PCR) for mutation

Submitted: March 1, 2017.

*Correspondence: E-mail: vladimir.shamanin@gmail.com Abbreviasions used: CT - computed tomography; EGFR - epidermal growth factor receptor; NSCLC - non-small cell lung cancer; $\mathrm{TKI}$ - tyrosine kinase inhibitors.
L858R and wild-type blocking real-time PCR for 19del (BioLink, Russia). Some rare deletions as well as insertions in exon 19 are not included in the real-time PCR assay, therefore we test for these mutations by fragment analysis of the DNA after PCR with forward primer 5'-dGGTGAGAAAGTTAAAATTCCCGTCGC and reverse primer 5'-dTCGAGGATTTCCTTGTTGGCTTTC. Fragment analysis of the DNA detected insertion in EGFR exon 19 (Fig. 2, a, case \# 692). Sanger sequencing of the DNA showed duplication of $18 \mathrm{bp}$ sequence AAAATTCCCGTCGCTATC (c.2215_2232dup) that resulted in mutation p.1744_K745insKIPVAI (Fig. 2, b).

During the first month after diagnosis the patient had 1 course of chemotherapy (carboplatin AUC5, etoposide $120 \mathrm{mg} / \mathrm{m}^{2}$ ). After detection of the EGFR mutation patient was switched to gefitinib $(250 \mathrm{mg} /$ day). One month after treatment with gefitinib there was pronounced improvement in clinical symptoms (minimal dyspnea at physical exercise, rare cough with small amount of sputum). Due to diffused infiltration of the lung parenchyma and pleuritis it was not possible to select target on CT scans for evaluation of response according to RECIST 1.1. Case was scored as partial response based on clinical improvements in patient performance from ECOG 2 to ECOGO- 1 and radiological improvements (diminished ground-glass opacities and decreased amount of fluid in pleural cavity on CT scans) after 2 months of treatment with gefitinib (Fig. 1, b). CT scans were performed every 2 months. Radiological and clinical disease progression was revealed after 12 months of gefitinib treatment. After that patient was switched to paclitaxel $\left(175 \mathrm{mg} / \mathrm{m}^{2}\right)$ and due to lack of clinical response after 3 courses of paclitaxel the best available supportive care was provided. Patient died 18 months after initiation of treatment. 


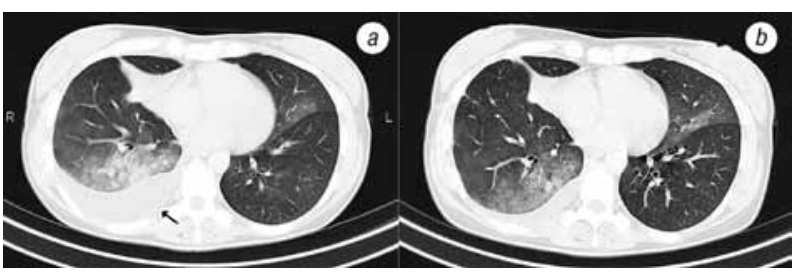

Fig. 1. CT scans of chest before (a) and after 2 months of treatment with gefitinib $(b)$. Note large amount of fluid in pleural cavity before treatment (arrow)

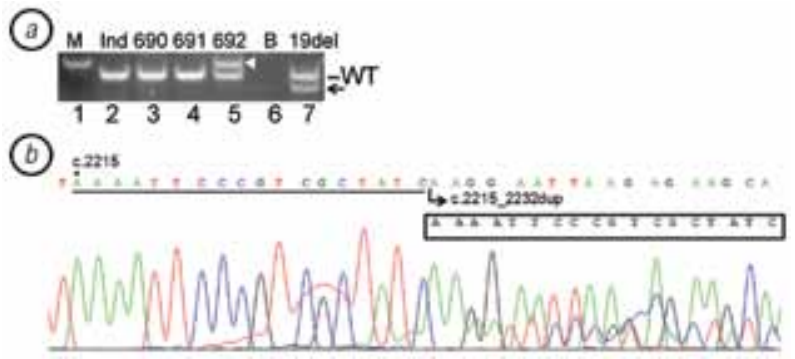

Fig. 2. Molecular analysis of a lung cancer case with insertion in EGFR exon 19. (a) Fragment analysis of EGFR exon 19. Picture of gel-electrophoresis of PCR DNA is shown with bands corresponding to exon 19 wild-type (WT), deletion (arrow) and insertion (arrow head). Lane 1 - 100bp DNA marker (M); lanes 2-5 DNA from FFPE tumor samples; lane 6 - blank (B); lane 7 - DNA standard with 15bp deletion in exon 19 (p.E746_A750delELREA). (b) Sanger sequencing of EGFR exon 19 in the tumor DNA\#692. Duplicated $18 \mathrm{bp}$ sequence in the tumor DNA is underlined, the insertion that resulted in mutation p.1744_K745insKIPVAI is shown in a box

\section{DISCUSSION}

In our laboratory the single 19 ins case was detected among 421 patients with NSCLC that included also 37 cases of $19 \mathrm{del}$ and 33 cases of L858R (Table). In another study of Russian patients with lung cancer 19ins was detected in 4 cases in addition to 455 cases with mutations 19del and L858R [3]. Frequency of 19ins among EGFR mutant cases in Russia based on lyevleva et al. [3] and our study together was $5 / 530(0.9 \%)$. This frequency is similar to $8 / 601$ (1.3\%) of cases with mutant EGFR in study in United States [2]. Interestingly, 19 ins comprised only $7 / 2581(0.3 \%)$ of the lung cancer cases in Asian patients with mutant EGFR [4, 5]. Much lower frequency of 19ins among Asian patients with mutant EGFR may be due to differences between ethnic groups or between methods to detect mutations in EGFR.

Table. Frequency of mutations EGFR L858R, 19del and 19ins in NSCLC in different studies

\begin{tabular}{|c|c|c|c|c|c|c|}
\hline $\begin{array}{l}\text { Patients, } \\
\mathrm{N}(\%)\end{array}$ & $\begin{array}{c}\mathrm{EGFR}^{+}, \mathrm{N} \\
(\%)^{1} \\
\end{array}$ & $\begin{array}{c}\text { 19del, N } \\
(\%)^{2}\end{array}$ & $\begin{array}{c}\text { L858R, N } \\
(\%)^{2}\end{array}$ & $\begin{array}{l}\text { 19ins, } \\
\mathrm{N}(\%)^{2} \\
\end{array}$ & $\begin{array}{c}\text { Coun- } \\
\text { try }\end{array}$ & Reference \\
\hline $\begin{array}{l}421 \\
(100.0)\end{array}$ & $71(16.9)$ & $37(52.1)$ & $33(46.5)$ & $1(1.4)$ & Russia & This study \\
\hline 276 & $459(20.2)$ & $288(62.7)$ & $167(36.4)$ & $4(0.9)$ & Russia & [3] \\
\hline $\begin{array}{l}3026 \\
(100.0)\end{array}$ & $601(19.9)$ & 347 (57.7) & $246(40.9)$ & $8(1.3)$ & USA & [2] \\
\hline $\begin{array}{l}2018 \\
(100.0) \\
3534\end{array}$ & $860(42.6)$ & na & na & $3(0.3)$ & $\begin{array}{l}\text { Hong } \\
\text { Kong }\end{array}$ & [4] \\
\hline 100.0) & $1721(48.7)$ & na & na & $4(0.2)$ & Taiwan & [5] \\
\hline
\end{tabular}

Note: ${ }^{1}$ mutant EGFR cases as percent of all patients; ${ }^{2}$ percent of cases with the mutation in comparison to all cases with mutant EGFR; na - not available.
Published data of response of lung cancers with 19ins to TKI (gefitinib, erlotinib or afatinib) includes 22 cases that were reviewed recently [5]. By searching PubMed in addition to the cases reviewed by Lin et al. [5] we found a report of Arabic female lung cancer patient with 19ins responding to erlotinib [6]. Together with our study total number of patients with 19ins treated with TKI is limited to 24 cases would-wide. Patients were predominantly female non-smokers and most cases were sensitive to TKI. We observed a case of lung adenocarcinoma (stage IV) with mutation p.1744_K745insKIPVAI that had partial response to gefitinib that lasted 12 months. Combined with data from lyevleva et al. [3] time to progression of Russian patients $(n=4)$ with 19ins on gefitinib was on average 9.3 months (range 5-12 months). For comparison, Caucasion patients who had lung cancer with mutant EGFR and were treated with gefitinib in the first-line had progression free survival 9.7 months [7]. It appears that sensitivity of tumors with 19ins and duration of response to TKI is similar to classic mutations in EGFR gene, however data on larger number of patients is needed.

In conclusion, our report contributes to small number of cases in other studies indicating sensitivity of the lung adenocarcinoma with insertions in exon 19 to therapy with TKI. The study also shows clinical usefulness of fragment analysis of EGFR exon 19 in addition to real-time PCR assays that do not include mutation 19ins.

\section{ACKNOWLEDGMENTS}

Theworkwassupported bygrant 15-14-10004 of Russian Scientific Foundation.

\section{DISCLOSURE}

The authors declare no conflict of interest.

\section{REFERENCES}

1. Mitsudomi T. Molecular epidemiology of lung cancer and geographic variations with special reference to EGFR mutations. Transl Lung Cancer Res 2014; 3: 205-11.

2. He M, Capelletti M, Nafa K, et al. EGFR exon 19 insertions: a new family of sensitizing EGFR mutations in lung adenocarcinoma. Clin Cancer Res 2012; 18: 1790-7.

3. Iyevleva AG, Mitiushkina NV, Karaseva NA, et al. Lung carcinomas with EGFR exon 19 insertions are sensitive to gefitinib treatment. J Thorac Oncol 2014; 9: e31-3.

4. Chan AW, Tong JH, Lo SH, et al. An uncommon insertion mutation in exon 19 of EGFR showed stable disease after TKI treatment. J Thorac Oncol 2013; 8: e107-8.

5. Lin YT, Liu YN, Wu SG, et al. Epidermal growth factor receptor tyrosine kinase inhibitor-sensitive exon 19 insertion and exon 20 insertion in patients with advanced non-small-cell lung cancer. Clin Lung Cancer 2017; 18: 324-32.

6. Agbarya A, Melamed-Frank M, Kaidar-Person O, et al. Getting out of a wheelchair: an uncommon insertion mutation in exon 19 of EGFR responsive to erlotinib. Springerplus 2014; 3: 507.

7. Douillard JY, Ostoros G, Cobo M, et al. First-line gefitinib in Caucasian EGFR mutation-positive NSCLC pa- 